\section{Time to Talk about Gender Equality in Math?}

Gender equality is always a hot topic, especially in the area of financial compensation. I recently was reminded of the importance of this issue when I interviewed the new chief executive officer of Microsoft, Satya Nadella, at the annual Grace Hopper Celebration of Women in Computing. Satya's response to "What advice would you give to women who feel uncomfortable in asking for a raise?" suggested that people should trust the system and that not asking for raises but simply doing good work would eventually result in rewards. Satya has since publicly apologized for those remarks. He said that his advice at the conference was "just plain wrong," despite being advice from his mentors that he has followed in his own career. He reinforced that this advice underestimated exclusion and bias - conscious and unconscious - that can hold people back in their careers. And, in fact, he has told all Microsoft employees that the company will work harder to make progress on diversity and inclusion at the core of Microsoft's culture in the areas of equal pay for equal work and equal opportunity for equal work, will recruit more diverse employees, and will expand training on how to foster an inclusive culture. This is real progress and action by Satya and Microsoft.

One of the positive outcomes of this recent experience is that it has sparked a valuable discussion about the importance of addressing gender equity issues with respect to pay, promotions, and access to resources. Despite numerous studies indicating that women are paid significantly less than men in most professions, the disparities remain. Among the hundreds of emails I received after the interview, one was from a female mathematician who wished that the mathematics community would have a serious discussion about gender equity issues in our profession. I'm hoping this "Opinion" piece might contribute to that discussion.

A very interesting article titled "Academic women in science, a changing landscape" was published recently by Stephen Ceci, et al. in Psychological Science. The article reviewed many recent studies on why there are so few female majors and faculty members in mathematically intensive areas of science and engineering and explores factors that might account for the difference in average pay received by men and women. I have personally been in situations where my discomfort in asking for a raise or negotiating a salary resulted in my being underpaid.
Am I the only mathematician who finds it easy to argue for raises, promotions, recognition, and resources for the people in my department but finds it difficult to ask for them for myself? Are there others who feel frustrated because they are always the only person who asks why there are so few women on lists of invited speakers or participants in prestigious events?

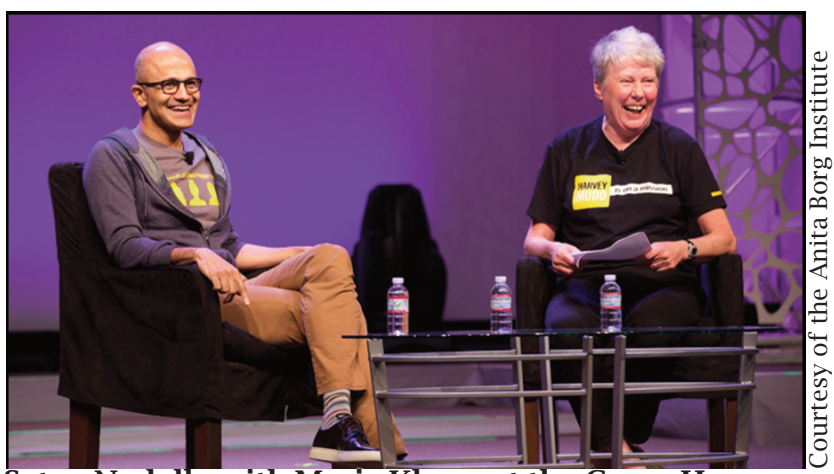

Satya Nadella with Maria Klawe at the Grace Hopper Celebration 2014.

And what about the high school teachers who still tell girls (but not boys) in their math and science classes they will have trouble with the more challenging concepts? There are many individuals and organizations who work on this every day, but isn't it time for all of us to work on it?

Satya said that when he became CEO, the advice he got was to be bold and be right. This advice I support, and I challenge all of us to apply this to our gender equality discussions and actions.

- Maria Klawe

Harvey Mudd College k7awe@hmc.edu 